\title{
DISORDERS OR RANGES OF HUMAN DIFFERENCE
}

\author{
John M. Richer \\ Department of Physiology, Anatomy and Genetics, University of Oxford, UK. \\ Paediatric Psychology, Oxford University Hospitals NHS Trust, UK. \\ johnricher@oxhs.co.uk
}

A Review of the Book

\section{Evolutionary Psychopathology: A unified approach}

By Marco del Giudice. 2018.

Oxford University Press. 560 pages.

ISBN 9780190246846 (Hardback, \$65.48, USD)

Marco del Giudice has set himself a daunting task to write a book describing an approach to psychiatric disorders which offers a fresh understanding, with overarching ideas informed by evolutionary theory. The scholarship is very impressive, there are nearly 2500 references stretching over almost 150 pages and to underpin his arguments. $\mathrm{He}$ brings together ideas from many fields of research: genetics, epigenetics, physiology, brain anatomy and function, epidemiology, psychology experiments and theories, psychiatric research and more.

The book starts with a brief tour of human evolution, and basic, but up to date, ideas within evolutionary theory: natural selection, gene selection, adaptation, conflict, e.g. parent offspring, sexual selection, mating strategies, group selection, cooperation, altruism and so on. The section on the "evolved mind" discusses various concepts such as motivations, executive function, and mentalising and mechanistic cognition. There is a useful summary of some key individual and sex differences. A major chapter is on life history theory and the distinction between fast and slow life histories. These concepts are applied in many of the later discussions. 
The second part of the book is a discussion of the major psychiatric disorders. Del Giudice very usefully summarises much research from various levels: genetic / physiological to psychological and epidemiological. He assesses the different explanatory theories on these various levels. He is careful to acknowledge the heterogeneity within a disorder, to report, or suggest, sub types based on data from the various research areas he reports, and to note where data fit or don't fit various theories. For each disorder he sets the discussion within an evolutionary framework looking at the possible adaptive advantages and disadvantages of the disorders or of their subclinical manifestations or of their connected traits in relatives.

It is worth quoting some of the useful unifying concepts he employs. In no particular order:

- Fast and slow life history strategies (already mentioned)

- Defense activation systems (strong in generalised anxiety disorder, phobias, depression, social anxiety, panic, avoidant personality disorder, etc.)

- Behavioural approach system (strong in mania) and behavioural inhibition system (strong in $\mathrm{ADHD}$ )

- The Big 5 personality traits, describing results from both diagnosed individuals, and from those who show related traits (e.g. schizotypy) and from family members

- Mentalistic vs mechanistic thinking - the diametrical model of autism (hyper mechanistic) and psychosis (hyper mentalistic)

The book is well written but nevertheless a difficult read due partly to the sheer density of empirical information, partly to the necessity to discuss on a fairly abstract level, and partly to the imprecision of psychiatric/psychological concepts that Del Giudice has little choice but to quote in reporting research findings. The difficulty of nailing down jelly springs to mind! But within the paradigm of evolutionary psychology/ psychiatry, this is a fine and scholarly book and rewards careful reading. My criticisms are not specifically of this book, but of the approach as a whole.

Psychologists and psychiatrists, with a few honourable exceptions (see for example the authors in Hutt, 1970), came late to embracing evolutionary theory, but, once they did, it added further scientific discipline and was a fertile source of ideas. These disciplines had always had approaches which embraced at least two of Tinbergen's four Whys? (Tinbergen, 1963), namely immediate causation and longer term causation, i.e. ontogeny. Also they had always looked at consequences of behaviour, but not, as ethologist would, asking about adaptive value within the context of evolutionary theory. Once evolutionary theory was "discovered" the four Whys? were, in effect, being asked. But...

What was still missing in much psychology was good direct observation of naturally occurring behaviour, and its description in non mentalistic terms. Tinbergen (1963) memorably wrote, "It has been said that, in its haste to step into the twentieth century and to become a respectable science, Psychology skipped the preliminary descriptive stage other natural sciences had gone through, and was soon losing touch with the natural phenomena". Ethology traditionally, and appropriately, demanded observation of 
the natural phenomena (i.e. phenomena uninfluenced or minimally influenced by the scientist, (Richer, 1974)). That provided a starting point of what needs to be understood. Without it, the would-be scientists do not know, literally, what they are talking about. Much psychology skipped this preliminary observation and adopted the so called "hypothetical deductive" method (e.g. Godfrey-Smith, 2003 p. 236, Haig, 2009), which emphasises the need to start with a hypothesis which is then tested. This method might be seen a welcome advance on mere assertion of a belief, but that sets the bar pretty low for judging an approach as scientific. It has been criticised in many ways, but crucially the question that is often not asked is, "from where does the hypothesis come, from what observable natural behaviour does it arise and what naturally occurring behaviour is it intended to help explain? The answer is rarely forthcoming and this is where the approach falls down - the research is not about the real publicly observable world, but about ideas derived from whatever is the psychologist's culture. Blurton Jones (1975) put this very succinctly when he said "the lateral thinking inductive approach of ethology may be contrasted with the deductive approach of psychology and its disdain for facts for their own sake" (page 72). Charlesworth (pers. comm.) puts it even more succinctly, if slightly cryptically: "follow the duck, not the theory of the duck".

The accusation of failing to observe the natural phenomena can be less fairly levelled at clinicians, who are confronted with it daily. Indeed they are privileged in being able to see first hand the wide range of human behaviour from the "hide" (c.f. birdwatcher's hide) of their clinical profession. Moreover they are doubly privileged in that daily they conduct Tinbergian "natural experiments", making small perturbations, (a.k.a. treatment) in the natural environment of their patients and observing the result (albeit perturbations determined by ethically wanting to help the patient, safely and efficaciously using well researched methods). But, despite this advantage....

The categories in which behaviour is described are often everyday ones, shot through with mentalistic assumptions, although the oddness of some of the behaviour of patients demanded, and got, a more objective approach to description. The diagnostic categories were developed to try and systematise and codify the problems brought to clinicians, and they have been helpful to an extent. But recently the category system of DSM-5 (APA 2013) been roundly criticised by many as being unfit for purpose (e.g. Richer, 2014). The diagnostic categories have been extensively criticised as not adequately helpful in either research or treatment. At best these categories roughly point to the sort of problem the patient has (which has some use).

This neglect of good direct observation leads to misdescription of behaviour. Two small examples from Del Giudice's book. Autistic children's often quoted insensitivity to pain is noted, but the actual observation of these children is that they often do not show the help-seeking or comfort-seeking behaviour or give obvious indication that they are "in pain". To assert the extreme hypothesis that the children do not feel pain, as some clinicians have done in the past, is nonsense since the children do not suffer the damage seen in children with, say, congenital analgesis. The children are avoiding communicating their pain. Another example is that the author uncritically quotes the diagnostic criteria for ADHD which, in the hyperactivity criterion, conflate displacement activities (fidgeting, fiddling, etc) with hyperactive behaviour (flitting from one activity to another). There are also some gaps, for instance, he quotes the idea of Pellicano and Burr (2012) of "bottom up" processing (from sensory input), versus "top down" processing 
(from expectations), and how the balance shifts to bottom up in autism. He does not make the obvious connection to the long established ideas of basic units of information processing where all action sets up expectations for feedback, and, as in Perceptual Control Theory (Powers 1973 ), or ordinary ideas about motivation, organisms act in order to achieve a certain input. Frith (2005) has interestingly suggested that a breakdown in this ability to expect feedback from actions underlies hallucinations and delusions, he argues, a person's own thoughts, perceptions and actions are sometimes seen as emanating from outside and not from the person himself. The gap in the book, as in evolutionary psychology and psychiatry, is the failure to describe the actual behaviour, and look at the immediate / proximate organising mechanisms in an effort to begin to understand that behaviour. Let me not minimise the difficulty of such an exercise, there are many impediments to doing such observation which I and others have detailed elsewhere (Richer, 2017).

In the second part of his book Del Giudice discusses the major diagnostic categories in turn. This is understandable for at least two reasons: (i) this is how his likely readership thinks and so Del Giudice will communicate more effectively and to a wide audience, (ii) these are the categories used in most research in this field. But it is frustrating to read through chapter after chapter where he describes the heterogeneity and dimensionality of the diagnostic categories, with their lack of clear boundaries between disorder and not, but still talks about them as if they were useful categories, pointing to "real" underlying phenomena. He makes interesting efforts to see the underlying mechanisms, and how they are shared amongst categories, but he does not go the whole way and say, "well maybe these diagnostic categories are not coherent or useful and a different approach needs to be adopted". He comes gently closest to this in his discussion of personality disorders. This is his next book perhaps, having paved the way with this one.

What would a thoroughgoing ethologist do in this field? S/he would recognise that the diagnostic categories, and the very concept of "disorder", were tools of the job for practicing clinicians. They are influenced by cultural norms, and at times, and at worst, by principles which conflate disorder with current local moral (e.g. homosexuality) or political (e.g. some Soviet psychiatry) ideas. These diagnoses are not scientific categories, they just help the clinician do their job. They are "emic" not "etic" terms, to use Pike's distinction (when an anthropologist describes a culture this can be couched in the culture's terms, "emic" or the anthropologist's terms, "etic", the latter being what a scientist does.)

An ethologist would simply look at the range of naturally occurring human behaviour, describe it in non mentalistic terms, hypothesise about its underlying structure, e.g. motivational structure, and ask Tinbergen's 4 Whys? of the description. What any group / society / culture chose to call a "disorder", thereby inviting clinical attention, might be included in that description as part of the behaviour of the group. If a group had this "map" then what they chose to call "disorders" could (theoretically) be described in terms of the map. The treatment of whatever behaviour was the issue would be informed by a knowledge of it causes, consequences and ontogeny from this scientific map. 
The actual practice of research is unlikely to be as purist as this. In any society there will always be cross fertilisation / cross contamination between the onlooker science and insider practice. However, like many concepts, this purist position describes a goal against which to compare current ideas and approaches.

Many clinicians, in their thinking and practice seem to embrace this way of thinking. Del Giudice too, seems to come close to doing this on a number of occasions, but then pulls back into the well worn paths of thought of conventional psychiatry. But this is his next book perhaps, having paved the way with this fine one.

\section{ABOUT THE AUTHOR}

John Richer is a child clinical psychologist at the University of Oxford and the University Hospital. He has published numerous many peer reviewed journal articles, books and book chapters. His research interests focus include on the application of ethology to "disturbed" behaviour, especially in children, attachment, hyperactivity, autism and behaviour problems, consciousness, and nutritional effects on behaviour. He has worked with multinational companies on psychological issues and is frequently asked to offer advice to Family Courts.

\section{REFERENCES}

American Psychiatric Association (2013): Diagnostic and Statistical Manual of Mental Disorders, Fifth Edition. Arlington VA: American Psychiatric Association. DOI

Blurton Jones, N. G. (1975). Ethology, anthropology and childhood. In R. Fox (Ed.) A.S.A. Studies-Biosocial Anthropology. London, Dent.

Frith, C. D.(2005) The neural basis of hallucinations and delusions. C.R. Biologies, 328(2), 170-175. DOI

Godfrey-Smith, P. (2003) Theory and Reality. Chicago, University of Chicago Press. DOI

Haig, B. (2009) Methods: Evaluating explanatory theories. The Psychologist, 22, 948-951

Hutt, SJ. (1970) The Role of Behaviour Studies in Psychiatry. In: Behaviour Studies in Psychiatry. Hutt, S.J. and Hutt C (Eds.) Elmsford, New York, Pergamon.

Pellicano E. \& Burr D. (2012) When the world becomes "too real": A Bayesian explanation of autistic perception. Trends in Cognitive Sciences, 16(10), 504-510. DOI

Powers, W.T. (1973) Behaviour: the Control of Perception. Chicago, Aldine.

Richer, J.M. (1974). Direct Observation - a reply to Cooper et al.. Bulletin of the British Psychological Society, 27, 500-502.

Richer, JM (2014) "Disordered behaviour" Alternatives to DSM-5 from an ethological perspective. Human Ethology Bulletin - Proc. of the IV ISHE Summer Institute 29. 27-55

Richer, JM (2017) Direct Observation: Impediments and approaches. Human Ethology Bulletin 32(4): Special Issue - Why Behaviour Observation?: 6-14. DOI

Tinbergen, N. (1963) On the aims and methods of ethology. Zeitschrift für Tierpsychologie, 20(4), 410-33. DOI 